\title{
Pré-Natal de alto risco: dados sociodemográficos e intercorrências durante a gravidez
}

High-risk prenatal care: sociodemographic and intercurrent data during pregnancy

Pre-Natal de alto riesgo: datos sociodemográficos e intercurrencias durante el embarazo

Joyce Driely Carvalho Silva ${ }^{1}$, Francidalma Soares Sousa Carvalho Filha ${ }^{1}$, Marcus Vinicius da Rocha Santos da Silva ${ }^{1 *}$, Emília Assunção Carvalho Silva ${ }^{1}$, Janderson Castro dos Santos ${ }^{1}$.

\section{RESUMO}

Objetivo: Analisar as características sociodemográficas e as intercorrências ocorridas com gestantes em Pré-natal de Alto risco em um município situado no nordeste brasileiro. Métodos: Trata-se de uma pesquisa avaliativa, do tipo exploratória, com abordagem quantitativa. Realizada com 95 gestantes, no período de março a setembro de 2017. Os dados foram organizados em um programa estatístico (SPSS) em seguida tabulados, transformados em tabelas e posteriormente feita a análise e interpretação dos mesmos com base na literatura produzida sobre o tema. Resultados: Averiguou-se que a maioria das gestantes $(67,4 \%)$ está na faixa etária entre 25 e maior ou igual a 36 anos; $67,3 \%$ são casadas ou mantém união estável; $57,9 \%$ tinham ensino médio completo; $50,5 \%$ estavam desempregadas. As principais intercorrências identificadas foram: hipertensão arterial/DHEG (16,8\%) abortamento habitual (11,6\%), idade superior a 35 anos $(10,5 \%)$ ou inferior a 15 anos $(9,5 \%)$. Foi percebido que $91(95,8 \%)$ das gestantes tiveram acompanhamento com enfermeiro, $67(70,5 \%)$ com médico e $38(40 \%)$ com médico ultrassonografista. Conclusão: O principal motivo da presença das gestantes no pré-natal de alto risco foi hipertensão arterial que é reconhecida como uma das principais complicações que levam o risco as gestações, destacou-se também o abortamento habitual e idade superior a 35 anos. Notou-se ainda a insuficiência de profissionais na atenção a uma parte dessas pacientes, visto que essas gestantes necessitam de um acompanhamento multiprofissional.

Palavras-Chave: Assistência Pré-Natal, Gravidez de Alto Risco, Gestante.

\section{ABSTRACT}

Objective: To analyze the sociodemographic characteristics and the intercurrences occurring with pregnant women in a high-risk prenatal care in a city located in northeastern Brazil. Methods: This is an exploratory research, with a quantitative approach. It was carried out with 95 pregnant women from March to September 2017. The data were organized in a statistical program (SPSS), tabulated, transformed into tables and later analyzed and interpreted based on the literature produced on the subject. Results: It was found that the majority of pregnant women $(67.4 \%)$ are in the age group between 25 and greater than or equal to 36 years; $67.3 \%$ are married or have a stable marriage; $57.9 \%$ had completed high school; $50.5 \%$ were unemployed. The main intercurrences identified were: normal hypertension / DHEG (16.8\%), habitual abortion (11.6\%), age greater than 35 years $(10.5 \%)$ or less than 15 years $(9.5 \%)$. It was noticed that $91(95.8 \%)$ of the pregnant women had follow-up with a nurse, 67 (70.5\%) with a doctor and $38(40 \%)$ with an ultrasonographer. Conclusion: The main reason for the presence of pregnant women in high-risk prenatal care was arterial hypertension, which is recognized as one of the main complications that lead to the risk of gestation. It was also highlighted the habitual abortion and age over 35 years. It was also noticed the insufficiency of professionals in the attention to a part of these patients, since these pregnant women need a multiprofessional accompaniment.

Keywords: Prenatal Care, High Risk Pregnancy, Pregnant Woman.

\footnotetext{
${ }^{1}$ Centro Universitário de Ciências e Tecnologia do Maranhão (UniFacema). Caxias-MA, Brasil.
}

*E-mail: marcusvinicius.darocha@yahoo.com.br

SUBMETIDO EM: $3 / 2019 \quad$ ACEITO EM: 4/2019 ～～PUBLICADO EM: 5/2019 


\section{RESUMEN}

Objetivo: Analizar las características sociodemográficas y las intercurrencias ocurridas con gestantes en prenatal de alto riesgo en un municipio situado en el nordeste brasileño. Métodos: Se trata de una investigación evaluativa, del tipo exploratorio, con abordaje cuantitativo. Se realizó con 95 gestantes, en el período de marzo a septiembre de 2017. Los datos fueron organizados en un programa estadístico (SPSS) luego tabulados, transformados en tablas y posteriormente realizada el análisis e interpretación de los mismos con base en la literatura producida sobre el tema. Resultados: Se verificó que la mayoría de las gestantes (67,4\%) está en el grupo de edad entre 25 y mayor o igual a 36 años; El 67,3\% están casadas o mantienen una unión estable; El 57,9\% tenía enseñanza media completa; El 50,5\% estaban desempleadas. Las principales intercurrencias identificadas fueron: hipertensión arterial / DHEG (16,8\%) aborto habitual $(11,6 \%)$, edad superior a 35 años $(10,5 \%)$ o inferior a 15 años $(9,5 \%)$. Se percibió que $91(95,8 \%)$ de las gestantes tuvieron seguimiento con enfermero, $67(70,5 \%)$ con médico y 38 (40\%) con médico ultrasonográfico. Conclusión: El principal motivo de la presencia de las gestantes en el prenatal de alto riesgo fue hipertensión arterial que es reconocida como una de las principales complicaciones que llevan el riesgo a las gestaciones, se destacó también el aborto habitual y edad superior a 35 años. Se notó la insuficiencia de profesionales en la atención a una parte de esas pacientes, ya que esas gestantes necesitan un acompañamiento multiprofesional.

Palabras clave: Asistencia Pre-Natal, Embarazo de Alto Riesgo, Gestante.

\section{INTRODUÇÃO}

O período gestacional na vida das mulheres promove o desenvolvimento de uma nova função social: ser progenitora, o que também exige a adaptação a novos desafios e modo de vida. Porém, nem todas as gravidezes acontecem de maneira hígida, pois alguns fatores podem ocasionar agravos à saúde da mãe e/ou do concepto, levando a dificuldades no processo gestacional, deixando de ser um fenômeno fisiológico, exigindo dos profissionais de saúde habilidades específicas para lidarem com este momento e da gestante alguns cuidados especiais (SILVA MR, et al., 2013).

O período pré-natal é considerado a etapa inicial para o parto e nascimento seguros e humanizados, o qual exige dos profissionais envolvidos preparo, a partir da implementação de uma efetiva comunicação, além de envolver gestante e sua família, tornando-os protagonistas desse processo e colaborando na efetivação de atividades de educação em saúde, estimulando o autocuidado e o desempenho da autonomia (COSTA CS, et al., 2013).

Segundo Caldas DB et al. (2013), a assistência ao pré-natal pode ser caracterizada como um programa de assistência à gestante, historicamente realizado pela medicina e pela enfermagem, que busca prevenir, diagnosticar e tratar situações indesejáveis à gestação, ao parto e ao recém-nascido.

Diante disso, a assistência à mulher é realizada da seguinte forma: após a confirmação de gravidez, baseada na história, exame físico e nos testes laboratoriais, inicia-se a captação da gestante para o prénatal, mediante a solicitação de testes e exames, o preenchimento do SisPré-Natal, do cartão da gestante e prontuário, realiza-se a avaliação de risco gestacional ainda na Estratégia Saúde da Família (ESF), e, tão logo detectados riscos, encaminha-se a mulher ao serviço de pré-natal de alto risco, onde é executada a avaliação pela equipe médica. Enfatiza-se que o pré-natal de alto risco requer acompanhamento no hospital de referência, com manutenção de assistência mensal na unidade básica de saúde (BRASIL, 2012a).

O conhecimento acerca do perfil da gestante de alto risco pode favorecer o progresso de ações e de políticas públicas de saúde que possibilitem reduzir os altos índices de gestações de alto risco e mortalidade perinatais (ANJOS JC, et al., 2014). Para Cabral SA et al. (2018), é essencial ofertar uma assistência individualizada para cada gestante no decorrer do pré-natal, tendo em vista os fatores socioeconômicos, os obstétricos, hábitos de vida, aspectos nutricionais, entre outros, de maneira a construir um laço de confiança entre a mulher e os profissionais envolvidos para melhor ampará-las no enfrentamento das complicações que poderão surgir ao longo de todo esse ciclo, principalmente no âmbito do pré-natal de alto risco. 
De acordo com a Portaria do Ministério da Saúde de no 1.020 de 29/05/2013 (BRASIL, 2013), o atendimento à gestante de alto risco abrange os vários níveis de complexidade, de acordo com as especificidades de cada mulher. Ademais, é constituído por uma equipe interdisciplinar com os seguintes profissionais: Enfermeiro Obstetra, Técnico de Enfermagem, Psicólogo, Assistente Social, Farmacêutico, Nutricionista, Médico Anestesiologista, Médico Obstetra, Médico Pediatra.

No que diz respeito aos fatores de risco anteriores e no decorrer da gestação, são classificados pelo Ministério da Saúde em: características individuais e condições sociodemográficas desfavoráveis, história reprodutiva anterior, condições clínicas preexistentes e no decorrer da gravidez (exposição indevida ou acidental a fatores teratogênicos e as intercorrências clínicas) (BRASIL, 2012b).

Dessa maneira, é fundamental explorar o perfil das mulheres grávidas e discernir os determinantes de saúde que podem intervir no progresso saudável da gestação, visto que essa caracterização dará apoio à equipe de saúde para o desencadeamento de ações de promoção que poderão oferecer melhoria na qualidade de vida das gestantes, assim como propiciará a efetivação dos encaminhamentos em momento conveniente no decorrer da assistência pré-natal (RODRIGUES ET, et al., 2017).

Nessa perspectiva, este artigo objetiva analisar as características sociodemográficas e as intercorrências ocorridas com gestantes em Pré-natal de Alto risco em um município do nordeste brasileiro.

\section{MÉTODOS}

Trata-se de uma pesquisa avaliativa, do tipo exploratória, com abordagem quantitativa. O cenário desta investigação foi o Município de Caxias, situado na região leste do estado do Maranhão, a 374 quilômetros da capital São Luís, a 70 quilômetros da capital piauiense, Teresina. Para tanto, utilizaram-se como campos de pesquisa uma maternidade pública e um Centro de Especialidade em Assistência Materno Infantil situados no referido município. Ressalta-se que a escolha dos locais de estudo deveu-se ao fato de que ambos são referência de atenção ao pré-natal de alto risco no município.

As participantes da pesquisa foram 95 gestantes assistidas nos dois serviços supracitados, as quais aceitaram de livre e espontânea vontade participar da pesquisa e assinaram o Termo de Consentimento Livre e Esclarecido e/ou o termo de assentimento (na situação de gestantes menores de idade).

Sobre isso, menciona-se que os critérios de inclusão foram: ser gestante diagnosticada com alguma intercorrência que comprometesse sua saúde e/ou gestação, sendo, por isso, cadastrada e acompanhada pelo serviço ambulatorial de Pré-natal de alto risco na Maternidade e/ou no Centro de Especialidade supraditos, sem limite de idade e/ou comorbidade, e aceitar de livre e espontânea vontade participar da pesquisa, bem como assinar o Termo de Consentimento Livre e Esclarecido ou Termo de Assentimento. Foram excluídas do estudo as gestantes que não realizaram pré-natal de alto risco nestas instituições e/ou não apresentaram gravidez de risco comprovada mediante diagnóstico médico.

Quanto à coleta de dados, ocorreu no período de março a setembro de 2017, de modo que foi aplicado um formulário com perguntas fechadas às gestantes, no qual continha itens relacionados aos dados sociodemográficos (idade, estado civil, procedência, escolaridade e profissão) e dados do acompanhamento Pré-Natal (intercorrências que levaram as mulheres a serem encaminhadas para o serviço de pré-natal de alto risco e as informações referentes a quais profissionais assistiram as gestantes no decorrer do prénatal).

No que diz respeito à organização e análise dos dados, a partir da aplicação dos formulários às gestantes, foram tabulados e transformados em tabelas, para uma posterior análise e interpretação dos mesmos. Em seguida, de posse dos dados referentes à assistência à gestante no Pré-natal de risco, compôs-se um banco de dados, os quais foram digitados no software Statistical Package for the Social Sciences- SPSS (versão 20.0 for Windows).

Quanto aos aspectos éticos do estudo, foram seguidos os seguintes procedimentos: antes do início da aplicação do formulário, a participante tomou ciência do objetivo do estudo, do instrumento aplicado e da 
garantia do anonimato e sigilo dos dados. Ao concordar em participar do estudo, a entrevistada assinou o Termo de Consentimento Livre e Esclarecido e no caso da participante (gestante) menor o Termo de Assentimento Livre e Esclarecido. O estudo supracitado seguiu os preceitos éticos, foi submetido à Plataforma Brasil, e, em seguida, direcionado ao Comitê de Ética em Pesquisa (CEP) da Faculdade de Ciências e Tecnologia do Maranhão, sendo aprovado com números de Certificado de Apresentação para Apreciação Ética (CAAE) 63469616.2.0000.8007 e 1.919.242 de Parecer.

\section{RESULTADOS}

Os resultados estão apresentados em subitens referentes aos formulários aplicados às gestantes do prénatal de alto risco. Dentre as características sociais das gestantes de alto risco participantes desta pesquisa, averiguou-se que $64(67,4 \%)$ estão na faixa etária entre 25 e maior ou igual a 36 anos. No que diz respeito à situação conjugal, $67(67,3 \%)$ são casadas ou mantém união estável. Quanto à variável procedência, $73(76,8 \%)$ gestantes são provenientes da zona urbana e $22(23,2 \%)$ da zona rural da cidade e de outros municípios vizinhos. Em relação ao nível de escolaridade, 55 (57,9\%) mulheres possuíam ensino médio completo. Averiguou-se também que 48 (50,5\%) gestantes não possuíam nenhum vínculo empregatício (Tabela 1).

Tabela 1 - Dados relativos às características sociais das gestantes que realizam pré-natal de alto risco. Caxias-MA, 2017.

\begin{tabular}{|c|c|c|}
\hline Variáveis & $\mathbf{N}$ & $\%$ \\
\hline \multicolumn{3}{|l|}{ Faixa Etária } \\
\hline$\leq 15$ anos & 4 & 4,2 \\
\hline $16-20$ anos & 12 & 12,6 \\
\hline $21-24$ anos & 15 & 15,8 \\
\hline $25-30$ anos & 20 & 21,1 \\
\hline $31-35$ anos & 23 & 24,2 \\
\hline$\geq 36$ anos & 21 & 22,1 \\
\hline \multicolumn{3}{|l|}{ Estado Civil } \\
\hline Solteira & 29 & 30,5 \\
\hline Casada & 46 & 48,4 \\
\hline Viúva & 1 & 1,1 \\
\hline União Estável & 18 & 18,9 \\
\hline Outra & 1 & 1,1 \\
\hline \multicolumn{3}{|l|}{ Procedência } \\
\hline Caxias- Zona Urbana & 73 & 76,8 \\
\hline Caxias- Zona Rural & 11 & 11,6 \\
\hline Outro Município & 11 & 11,6 \\
\hline \multicolumn{3}{|l|}{ Escolaridade } \\
\hline Fundamental $1-1^{\underline{a}}$ a $4^{a}$ serie & 4 & 4,2 \\
\hline Fundamental II - $5^{\mathrm{a}}$ a $8^{\mathrm{a}}$ serie & 24 & 25,3 \\
\hline Médio incompleto & 12 & 12,6 \\
\hline Médio completo & 32 & 33,7 \\
\hline Superior incompleto & 16 & 16,8 \\
\hline Superior Completo & 7 & 7,4 \\
\hline \multicolumn{3}{|l|}{ Profissão } \\
\hline Autônoma & 8 & 8,4 \\
\hline Professora & 6 & 6,3 \\
\hline Técnica de enfermagem & 3 & 3,2 \\
\hline Lavradora & 17 & 17,9 \\
\hline Outra & 19 & 13,7 \\
\hline Nenhuma & 48 & 50,5 \\
\hline Total & 95 & 100,0 \\
\hline
\end{tabular}

Fonte: Dados da pesquisa, 2017. 
Em relação às intercorrências que provocaram os diagnósticos de gestação de alto risco nas gestantes participantes do estudo, as quatro mais representativas foram hipertensão arterial/DHEG, com $16(16,8 \%)$ casos, abortamento habitual com $11(11,6 \%)$, idade superior a 35 anos sendo representada por $10(10,5 \%)$ casos e $9(9,5 \%)$ gestantes com idade inferior a 15 anos. Quanto aos profissionais que prestaram assistência às mulheres participantes da pesquisa durante o pré-natal, foi percebido que 91 (95,8\%) gestantes foram acompanhadas por enfermeiro, 67 (70,5\%) por médico e 38 (40\%) por médico ultrassonografista (Tabela 2).

Tabela 2 - Dados referentes as intercorrências que levaram as gestantes a serem encaminhadas ao prénatal de alto risco e os profissionais que prestaram assistência. Caxias-MA, 2017.

\begin{tabular}{|c|c|c|}
\hline Variáveis & $\mathbf{N}$ & $\%$ \\
\hline \multicolumn{3}{|l|}{ Intercorrências } \\
\hline Gestação múltipla & 4 & 4,2 \\
\hline Hemorragias & 4 & 4,2 \\
\hline Diabetes/diabetes gestacional & 3 & 3,2 \\
\hline Hipertensão Arterial/DHEG & 16 & 16,8 \\
\hline Anemia & 3 & 3,2 \\
\hline Abortamento habitual & 11 & 11,6 \\
\hline Fator Rh negativo da mãe & 3 & 3,2 \\
\hline Pré-eclâmpsia/Eclâmpsia & 3 & 3,2 \\
\hline Idade Inferior a 15 & 9 & 9,5 \\
\hline Idade Superior a 35 & 10 & 10,5 \\
\hline Asma & 3 & 3,2 \\
\hline Depressão & 3 & 3,2 \\
\hline Complicações na gestação anterior & 3 & 3,2 \\
\hline Cesária Habitual & 3 & 3,2 \\
\hline Outras & 26 & 27,5 \\
\hline Ignorado & 3 & 3,2 \\
\hline \multicolumn{3}{|l|}{ Profissionais } \\
\hline Médico & 67 & 70,5 \\
\hline Enfermeiro & 91 & 95,8 \\
\hline Assistente social & 14 & 14,7 \\
\hline Psicólogo & 19 & 20,0 \\
\hline Nutricionista & 3 & 3,2 \\
\hline Endocrinologista & 1 & 1,1 \\
\hline Ultrassonografista & 38 & 40,0 \\
\hline Total & 95 & $100, \mathrm{C}$ \\
\hline
\end{tabular}

Fonte: Dados da pesquisa, 2017.

\section{DISCUSSÃO}

No que tange às características sociais das participantes, quanto à faixa etária, observou-se que a maioria estava com idade entre 25 e maior ou igual a 36 anos. Desse modo, nota-se que a idade supostamente foi uma condição importante para o risco gestacional. Relacionado a isso, em um estudo caso-controle sobre fatores de risco para mortalidade materna, desenvolvido por Leite RM et al. (2011) em Recife, foi apurado que mulheres de 20 a 34 anos de idade prevaleceram entre os casos, considerando-se que nessa faixa etária há o maior número de gestações, sendo que com a idade aumenta-se o risco de morte, acentuando-se bastante a partir dos 35 anos ou mais.

Em termos comparativos, convém citar o estudo realizado por Costa LD et al. (2016), em um Instituto da Mulher, que avaliou prontuários de gestantes de alto risco e encontrou predomínio da faixa etária de 15 a 35 anos (82\%). Além disso, averiguação realizada por Gravena AA et al. (2012) verificou que as mulheres com idade superior a 35 anos apresentam maior frequência de resultados perinatais desfavoráveis quando 
comparadas com as mulheres com idade entre 20 e 34 anos. Logo, a gestação tardia necessita de cuidado obstétrico rigoroso, principalmente se houver alguma patologia associada. Este processo de referenciamento de mulheres gestantes a vários níveis de complexidade e especialidade deve proporcionar o acesso aos serviços de saúde, segundo suas necessidades, suscetibilidades e problemas de saúde.

Sobre a situação conjugal, evidenciou-se um dado favorável para o bom desenvolvimento do pré-natal, haja vista que, teoricamente, esta mulher teria o apoio de um(a) parceiro(a) para dividir angústias, anseios e até dúvidas e medos. Ressalta-se que estes resultados coincidem com outros estudos, assim como o de Rodrigues ET (2010), o qual verificou na sua amostra que, quanto à situação conjugal, $59,1 \%$ das gestantes apresentavam estado civil de casada ou mantinham união estável.

No que diz respeito à variável procedência, mais da metade das gestantes é proveniente da zona urbana e somente $23,2 \%$ da zona rural da cidade e de outros municípios vizinhos. No que tange à variável escolaridade, evidenciou-se que uma parcela considerável das participantes concluíram o ensino médio ou possuem mais de doze anos de estudos, algo que se apresenta como um resultado positivo e que não estaria diretamente ligado a um fator de risco. Sobre isto, o Ministério da Saúde (BRASIL, 2004), alega que a baixa escolaridade é avaliada como fator perigoso para as gestantes, pois à proporção que o nível escolar diminui, percebe-se a redução e/ou ausência de conhecimento e de informações acerca das condições de funcionamento do próprio corpo e da intervenção do meio ambiente no estabelecimento da própria saúde.

Ratificando tais informações, Sousa JJ (2011) ressalta que o nível de escolaridade é uma causa que afeta de forma direta no momento de decisão sobre uma gravidez ser planejada ou não. Por isso, a carência de informação e conhecimentos sobre a sexualidade e reprodução, utilização adequada de métodos contraceptivos e a baixa presença masculina na contracepção precisam ser levados em consideração para que assim o sistema de saúde opere por meio de estratégias competentes para modificar esse cenário social existente.

O estudo revelou que metade das participantes não possuía nenhuma ocupação, tratando-se de um resultado elevado, o que poderia se configurar como um fator predisponente ao risco, além disso, dentre as que trabalhavam, predominam profissões pouco especializadas e com baixos salários, o que contribui para a manutenção das condições socioeconômicas precárias. Referente a esta variável, investigação desenvolvida por Marques AG et al. (2012), constituída por informações obtidas de fichas obstétricas de gestantes atendidas por enfermeiras no Programa de Saúde da Mulher no período de 1972 a 2009, apresenta semelhança com o presente estudo, na qual metade das participantes $(51,1 \%)$ também não apresentavam nenhum vínculo empregatício.

No que concerne à problemática das intercorrências, enfatiza-se que a Hipertensão Arterial é considerada como uma das mais significativas adversidades do período gestacional e puerperal, recebendo, inclusive, denominação própria neste período, Doença Hipertensiva Específica da Gestação (DHEG), estando diretamente ligada ao resultado do alto risco de morbidade e mortalidade materna e perinatal, já que a partir da mesma podem surgir outras comorbidades com caraterísticas mais severas, como a préeclâmpsia ou eclâmpsia, ou até mesmo uma complicação mais extrema, que é o caso da chamada Síndrome de Hellp, um conjunto de alterações que cursa com hemólise, aumento das enzimas hepáticas e diminuição de plaquetas.

Assim, acompanhar as gestantes, considerando as interferências desses fatores para a evolução da gravidez, colabora para intervir precocemente, proporcionando um atendimento resolutivo frente a esses sinais de alerta, pois dentre os diagnósticos levantados no estudo notou-se a predominância da hipertensão arterial sistêmica ou DHEG e do abortamento habitual, que são causas passíveis de controlar por meio da implementação do tratamento medicamentoso, alimentação saudável, dieta adequada, repouso, dentre outras ações.

Sobre esse assunto, convém evidenciar os achados de uma investigação desenvolvida por Rodrigues ET (2010), na qual houve análise de prontuários de 159 gestantes de alto risco internadas no Hospital Regional de Cáceres Dr. Antonio Fontes, entre os anos de 2009 a 2010, e ficou demonstrado que dentre os 
diagnósticos detectados na gravidez atual, os principais eram doença hipertensiva específica da gravidez e trabalho de parto prematuro, ambos com a mesma porcentagem de $34,6 \%$.

Para Correia DS et al. (2011), os riscos da gestação geralmente estão relacionados a aspectos psicológicos tais como: ausência de apoio familiar, níveis elevados de estresse, presença de sintomas depressivos e baixa expectativa relacionada ao futuro. Destaca-se também que a presença do estresse pode influenciar na relação da mãe com seu bebê, a exemplo da elevação de ocorrência de maus tratos em filhos, quando a gravidez não é planejada.

Nesta perspectiva, salienta-se que as gestantes carecem de uma assistência competente para que as intercorrências dessa fase sejam evitadas e prevenidas ou pelo menos amenizadas, com vistas a se proporcionar formas mais coerentes de parir e de nascer. Desse modo, a equipe atuante necessita estar inteirada do perfil das clientes que irão acolher, propiciando a implementação de estratégias particulares e pertinentes que assegurem a eficácia do serviço prestado.

No que diz respeito aos profissionais que prestaram assistência às mulheres participantes da pesquisa durante o pré-natal, foi percebido que a maioria teve acompanhamento com enfermeiro, médico e menos da metade com médico ultrassonografista. Constatou-se também que houve pouca demanda em relação aos demais profissionais, o que poderia ser justificado pela falta destes em um dos locais onde foi desenvolvido o estudo.

Quanto a isso, Barbosa TL et al. (2011) explicitam que o(a) enfermeiro(a) se apresenta como membro dinâmico dos trabalhadores de saúde, por desempenhar uma função educativa e colaborar para a ocorrência de mudanças concretas e saudáveis nas atitudes das gestantes, dos familiares e da comunidade, sempre em busca de bem-estar e qualidade de vida das pessoas assistidas.

\section{CONCLUSÃO}

Ao analisar os dados sociodemográficos e as intercorrências das gestantes de alto risco, conclui-se que as gestantes apresentam perfis comuns a participantes de outros estudos. O principal motivo da presença das gestantes no pré-natal de alto risco foi hipertensão arterial, que é reconhecida como uma das principais complicações que implica risco às gestações. Destacaram-se também outras complicações, como: abortamento habitual e idade superior a 35 anos. Notou-se ainda a insuficiência de profissionais na atenção a parte dessas pacientes, visto que essas gestantes necessitam de um acompanhamento multiprofissional. Entende-se que, indubitavelmente, a gravidez de alto risco necessita de cuidados mais complexos, pois existem diversos fatores que fragilizam a gestante e a tornam vulnerável à intercorrências durante o período gravídico. Vale ressaltar que o alcance de uma assistência de qualidade ao pré-natal de alto risco requer o desenvolvimento de melhorias dos indicadores de saúde perinatal e a definição rigorosa do perfil epidemiológico das gestantes assistidas nos sistemas de saúde.

\section{REFERÊNCIAS}

1. ANJOS JCS, et al. Perfil epidemiológico das gestantes atendidas em um centro de referência em pré-natal de alto risco. Revista Paraense de Medicina, 2014; 28 (2):23-33.

2. BARBOSA TLA, et al. O pré-natal realizado pelo enfermeiro: a satisfação das gestantes. Cogitare Enferm, $2011 ; 16(1): 29-35$.

3. BRASIL. Ministério da Saúde. Secretaria de Atenção à Saúde. Departamento de Ações Programáticas Estratégicas. Política nacional de atenção integral à saúde da mulher: princípios e diretrizes. Brasília: $\begin{array}{lllll}\text { Ministério da } \quad \text { Saúde. } & \text { [s.n.: } & \text { s.l.], } & & \end{array}$ http://bvsms.saude.gov.br/bvs/publicacoes/politica_nac_atencao_mulher.pdf. Acesso em: 22 out. 2018.

4. Ministério da Saúde. Secretaria de Atenção à Saúde. Departamento de Ações Programáticas Estratégicas. Atenção ao pré-natal de baixo risco. Brasília: Ministério da Saúde. [s.n.: s.l.], 2012a. Disponível em: http://bvsms.saude.gov.br/bvs/publicacoes/cadernos_atencao_basica_32_prenatal.pdf. Acesso em: 22 out. 2018. 
5. Ministério da Saúde. Secretaria de Atenção à Saúde. Departamento de Ações Programáticas Estratégicas. Manual Técnico de Gestação de Alto Risco. Brasília: Ministério da Saúde. [s.n.: s.l.], 2012b. Disponível em: bvsms.saude.gov.br/bvs/publicacoes/gestacao_alto_risco.pdf . Acesso em: 22 out. 2018.

6. Ministério da Saúde. Portaria no 1.020 de 29 de maio de 2013. Atenção à Saúde na Gestação de Alto Risco. Brasília: Ministério da Saúde. [s.n.: s.l.], 2013. Disponível em: http://bvsms.saude.gov.br/bvs/saudelegis/gm/2013/prt1020_29_05_2013.html. Acesso em: 22 out. 2018.

7. CABRAL SAAO, et al. Receios na gestação de alto risco: uma análise da percepção das gestantes no prénatal. Rev Mult Psic, 2018; 12(40): 151-162.

8. CALDAS DB, et al. O Atendimento psicológico no pré-natal de alto risco: a construção de um serviço. Psic Hosp, 2013; 11(1):66-87.

9. CORREIA DS, et al. Adolescentes grávidas: sinais, sintomas, intercorrências e presença de estresse. Rev Gaúcha Enferm, 2011; 32(1): 40-47.

10. COSTA CSC, et al. Características do atendimento pré-natal na Rede Básica de Saúde. Rev Eletr Enferm, 2013; 15(2): 516-522.

11. COSTA LD, et al. Perfil epidemiológico de gestantes de alto risco. Rev Cogitare Enferm, 2016; 21(2):01-08.

12. GRAVENA AAF, et al. Resultados perinatais em gestações tardias. Rev Esc Enferm USP, 2012; 46(1):1521.

13. LEITE RMB, et al. Fatores de risco para mortalidade materna em área urbana do Nordeste do Brasil. Cad Saúde Pública, 2011; 27(10):1977-1985.

14. MARQUES AGB, et al. Características de gestantes atendidas em consulta de enfermagem ambulatorial de pré-natal: comparação de quatro décadas. Rev Gaúcha Enferm, 2012; 33(4):41-47.

15. 15-RODRIGUES ET. Perfil epidemiológico das gestantes de alto risco internadas em um hospital de Cáceres - Mato Grosso. Dissertação (Mestrado). Universidade de Brasília, Brasília, 2010; $128 \mathrm{p}$.

16. 16-RODRIGUES ARM, et al. Gravidez de alto risco: análise dos determinantes de saúde. Sanare, 2017; 16(1):23-28.

17. 17-SILVA MRC, et al. A percepção de gestantes de alto risco acerca do processo de hospitalização. Rev Enferm UERJ, 2013; 21(2): 792-797.

18. 18-SOUSA JJ. Circunstâncias da ocorrência de gravidez não planejada em mulheres adultas. Dissertação (Mestrado). Escola de Enfermagem da Universidade Federal da Bahia, Salvador, 2011; 119 p. 